\title{
A Secondary Source Configuration for Control of a Ventilation Fan Noise in Ducts
}

\author{
Jurij Prezelj* - Mirko Čudina \\ University of Ljubljana, Faculty of Mechanical Engineering, Slovenia
}

The main noise source in heating, ventilation, and air conditioning systems is usually a ventilating fan. Noise, generated by the ventilating fan is transmitted through the duct into the living and working environment. A typical fan noise spectrum consists of a broadband noise, which is superimposed with pure tones. Different methods are available to reduce a transmission of such noise from the ventilating fan into the living and working environment. In this article it is demonstrated how a feedforward active noise control system can be implemented together with a side branch resonator. Effectiveness of the feedforward active noise control system depends on the quality of a reference signal, which should be in a perfect correlation with the primary noise. An acoustic feedback is the main problem of feedforward active noise control systems in ducts. A combined method uses a single loudspeaker to work as a dipole source and a side branch resonator to reduce the acoustic feedback. A side branch resonator reduces noise transmission in a narrowband frequency range as well. In this article, a theoretical background of a dipole source with a side branch resonator is presented, along with some measurement results and simulations of active noise control.

(C) 2011 Journal of Mechanical Engineering. All rights reserved.

Keywords: active noise control, secondary source, ventilation duct, noise, fan

\section{INTRODUCTION}

Noise generated by heating, ventilating, and air conditioning (HVAC) systems, is a combination of narrowband discrete frequencies and a broadband random noise. Narrowband discrete frequencies are correlated with a blade passage frequency from the ventilating fan, and an electromagnetic noise, which is correlated to a mains frequency (50 or $60 \mathrm{~Hz}$ ), and higher harmonics. A broadband random noise is aeroacoustically generated by an intensive turbulent air flow. Several different types of silencers can be used to reduce noise propagation through HVAC systems. A reduction of noise propagation through the duct with a side branch resonator is a wellknown method [1] and [2]. With this method a significant reduction of noise propagation can be achieved, but only in a narrow frequency bands. Active noise control (ANC) is an attractive method, which can be used for a narrowband and/ or a broadband noise control in a low frequency range. A noise-cancellation speaker emits a sound wave with the same amplitude but with an inverted phase to the primary noise. The waves combine to form a new wave, in a process called interference, and effectively cancel each other out. Effectiveness of the feedforward ANC system depends on the quality of the reference signal, which should be in a perfect correlation with the primary noise. An acoustic feedback is the main problem of the feedforward ANC systems in ducts. In the available literature, only a few studies discuss the problem of reducing the acoustic feedback in the acoustical domain, as for example [3] to [5]. A method, which combines a single loudspeaker to work as a dipole secondary source with a side branch resonator to reduce the acoustic feedback, is presented in this article. A basic depiction is given in Fig. 1. A basic Swinbanks theory [6] was used to explain how a dipole source combined with a side branch resonator forms a unidirectional secondary source for the ANC system. Swinbanks showed that two monopole sources with appropriate signal delay form a unidirectional sound source in the duct. The source strength of the upstream monopole at the time $t$ is $q_{1}(t)$ and that of the downstream source is $q_{2}(t)$. There will be zero output from the combination of the two monopole sources in the upstream direction if: 


$$
q_{1}(t)=-q_{2}\left(t-\frac{L}{c_{0}(1-M)}\right) .
$$

In Eq. (1) $L$ denotes the spacing between two monopole sources (Fig. 1), $c_{0}$ is the speed of sound and $M$ is the Mach number of net airflow. If the speed of the airflow through the duct is less than $3 \mathrm{~m} / \mathrm{s}$, it has practically no influence on time delay settings, [7] and [8]. From Eq. (1) it can be derived that $q_{1}$ must have the same amplitude as $q_{2}$, but it must be inverted and delayed relative to $q_{2}$ by $L / c_{0}$ in order to minimize acoustic feedback. A time delay $\tau_{0}$ should be equal to the time, which is needed for "anti-noise" to travel from the downstream source to the upstream source. This distance is marked with $L$ and basically represents the length of the side branch resonator.

$$
\tau_{0}=\frac{L}{c_{0}(1-M)} \text {. }
$$

To satisfy conditions in Eqs. (1) and (2), two monopole sources with the same amplitude and reverse phase have to be provided. Two sets of loudspeakers with two different amplifiers and a unit for adding appropriate time delay into the signal are typically used. The basic idea of the proposed design is to use sound, which is generated on the backside of the loudspeaker cone, for acoustic feedback reduction [10]. Sound from the front side of the loudspeaker, which is propagating upstream, is cancelled by the sound generated on the rear side of the loudspeaker. An acoustical short circuit is used for acoustic feedback reduction and the side branch resonator (SBR) acts like a transmission line for frequency response improvement of the secondary source in band pass frequency range. Swinbanks method is actually transferred into the pure acoustic domain, Fig. 2 [11]. Dipole source can also be used for improvement of the insertion loss on walls. Insertion loses can be improved by using dipole sources, however, a performance of the ANC system with a dipole source significantly depends on the acoustic setup [12]. A systematic analysis of the acoustic setup is therefore needed.

A block chart is presented in Fig. 2 and it explains a proposed system. $P(z)$ denotes a primary acoustic path from the reference microphone position to the error microphone position. $C(z)$ denotes a transfer function of all controller elements (microphone, AD/DA converter, preamplifier, compensation filters, and power amplifier); $S_{F}(z)$ presents a transfer function of sound radiation from the loudspeaker front side, including the acoustic path to the error microphone position; $S_{S B R}(z)$ presents a transfer function of sound radiation from the loudspeaker rear side, including the acoustic path through the side branch resonator and through the main duct to the error microphone position; $F_{F}(z)$ presents a transfer function of sound radiation from the loudspeaker front side, including the acoustic path to the reference microphone position through the main duct; $F_{S B R}(z)$ presents a transfer function of sound radiation from the loudspeaker rear side, including the acoustic path through the side branch resonator to the reference microphone position. The negative sign denotes an inversed phase between the loudspeaker rear side and front side sound radiation.

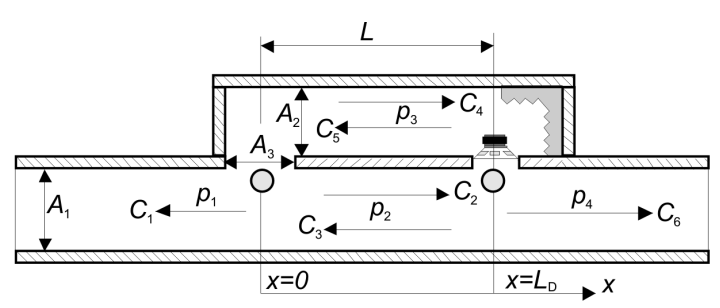

Fig. 1. A dipole source in a side branch resonator

From the block chart it can be deduced that a transfer function of sound generation at the front side of the loudspeaker must be equal to the transfer function of sound generation at the rear side of the loudspeaker including acoustic paths $\left(F_{S B R}(z)=F_{F}(z)\right)$, in order to minimize the acoustic feedback. A typical loudspeaker is a perfect dipole source in the discussed frequency range. Only the geometrical configuration of the secondary source affects the properties of two acoustical paths and consequently the acoustic feedback. Therefore, a special attention should be paid to the geometry design of the secondary source.

\section{ONE DIMENSIONAL LINEAR MODEL}

An additional acoustical element erected near the secondary source (loudspeaker), affects the impulse response of the ANC system in both duct directions. A frequency response of the new 


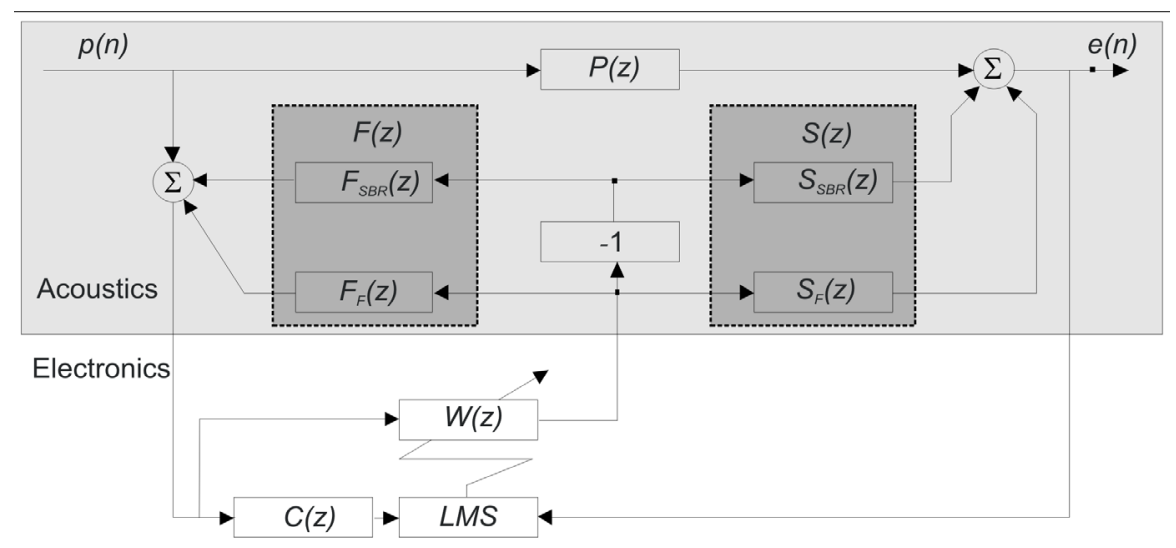

Fig. 2. A block chart of the proposed feedforward ANC configuration

secondary source configuration is not easy to determine due to some factors in the acoustical domain, which have to be taken into consideration. The frequency response of the proposed secondary source configuration strongly depends on the geometry of the setup. When combining the SBR with a dipole source, some parameters have to be carefully considered:

- the position of the loudspeaker in the SBR $\left(L_{D}\right)$ relative to the SBR opening,

- the length of the SBR $(L)$,

- the ratio between a SBR cross section $\left(A_{2}\right)$ and the main duct cross section $\left(A_{1}\right)$,

- the ratio between a SBR opening $\left(A_{3}\right)$ and the main duct cross section $\left(A_{1}\right)$, and

- damping in the SBR $(\alpha)$.

ANC systems are used for noise control in the low frequency range. A theoretical model was therefore developed for one-dimensional wave propagation in an infinite duct. A proposed configuration of the secondary source is presented in Fig. 1. In such configuration four sound pressure fields $\left(p_{1}, p_{2}, p_{3}\right.$ and $\left.p_{4}\right)$ and four related velocity fields $\left(u_{1}, u_{2}, u_{3}\right.$ and $\left.u_{4}\right)$ are developed. Each pressure field is described by one wave equation. A sound pressure field denoted with $p_{1}$, presents a wave propagation from the SBR opening, back to the primary noise source $(x=0)$. Since there is no propagation of sound pressure from the left side of the duct, a solution of the wave equation, Eq. (4) has only one part. $C_{1}$ presents the amplitude of sound that is propagating in the upstream direction. $C_{1}$ presents the acoustic feedback and its value should be as low as possible. Both parts of the solution are included in equations for sound pressure field $p_{2}$ and for $p_{3}$. A standing wave is formed in the main and in the side channel between loudspeaker $\left(x=L_{D}\right)$ and opening $(x=0)$. A Sound pressure field denoted with $p_{4}$ presents a useful part of sound generated by the secondary source in the downstream direction. $C_{6}$ should be larger than $C_{1}$ in order to achieve directivity and consequently, a reduction of the acoustic feedback.

Six unknowns $\left(C_{1}\right.$ to $\left.C_{6}\right)$ describe the amplitude of four sound fields. Six independent boundary conditions must be fulfilled in order to solve the system. Boundary conditions are known at two locations in the duct, denoted with a gray spot at $x=0$ and at $x=L$ (Fig. 1).

A first set of boundary conditions is given at the SBR opening $(x=0)$. At this position two channels of the secondary source are merged and a cross section of the main duct is changed. Sound pressure of three sound fields at this point must be the same in order to prevent net flow due to the sound pressure field. Conservation of mass flow at this point $(x=0)$ can be used as a third boundary condition. A second set of known boundary conditions can be obtained at the loudspeaker position $(x=L)$. A loudspeaker is regarded as a moving surface $S$ with a known surface velocity. Volume flow at the backside of a loudspeaker cone is exactly the same as the volume flow on the front side of the loudspeaker cone. Volume flow generated on the backside of the loudspeaker cone is distributed across SBR cross section $\left(A_{2}\right)$. Volume flow generated at the front side of the loudspeaker cone is distributed in two directions of the main duct $\left(2 A_{1}\right)$. In Eqs. (7) and (8), $u_{0}$ represents a surface velocity of 
the loudspeaker cone. Sound pressure to the right of the loudspeaker is exactly the same as sound pressure to the left of the loudspeaker at the front side of loudspeaker $(x=L)$ in the main duct. These six boundary conditions are given in Eqs. (3) to (8):

$$
\begin{gathered}
p_{1}(x=0)=p_{2}(x=0), \\
p_{2}(x=0)=p_{3}(x=0), \\
A_{1} u_{1}(x=0)+A_{2} u_{3}(x=0)+A_{1} u_{2}(x=0)=0, \\
p_{2}(x=L)=p_{4}(x=L) \\
A_{2} u_{3}(x=L)=S v_{0} \\
A_{1} u_{2}(x=L)+S v_{0}=A_{1} u_{4}(x=L) .
\end{gathered}
$$

Coefficients $C_{1}$ and $C_{6}$ present the amplitude of sound generated by the secondary source in two opposite directions. $C_{1}$ presents a part of the sound field, which propagates into a negative direction towards the reference microphone and towards the primary noise source. Coefficient $C_{6}$ presents a useful part of the sound field, which is propagating in a positive direction and is used for noise control.

These two coefficients can be simply obtained by solving the linear system given in Eq. (9). The results are given in Eqs. (10) and (11).

$$
\begin{gathered}
{\left[\begin{array}{cccccc}
1 & -1 & -1 & 0 & 0 & 0 \\
1 & 0 & 0 & -1 & -1 & 0 \\
-A_{1} & -A_{1} & A_{1} & -A_{2} & A_{2} & 0 \\
0 & e^{i k L} & e^{-i k L} & 0 & 0 & -e^{i k L} \\
0 & 0 & 0 & A_{2} e^{i k L} & -A_{2} e^{-i k L} & 0 \\
0 & A_{1} e^{i k L} & -A_{1} e^{-i k L} & 0 & 0 & -A_{1} e^{i k L}
\end{array}\right]\left[\begin{array}{l}
C_{1} \\
C_{2} \\
C_{3} \\
C_{4} \\
C_{5} \\
C_{6}
\end{array}\right]=} \\
=\left[\begin{array}{c}
0 \\
0 \\
0 \\
0 \\
\rho c u_{0} S \\
-\rho c u_{0} S
\end{array}\right] . \\
C_{1}=-\rho c u_{0} S \frac{1}{A_{2}}\left(\frac{e^{-i k L}-e^{i k L}}{3+e^{2 i k L}}\right) \\
C_{6}=\rho c v_{0} S\left(\frac{1}{A_{1}}+\frac{1}{A_{2}}\right)\left(\frac{e^{-i k L}-e^{i k L}}{3+e^{2 i k L}}\right)
\end{gathered}
$$

Coefficients $C_{1}$ and $C_{6}$ depend on the $k L$ value. Coefficients $C_{1}$ and $C_{6}$ also depend on the ratio between the main duct cross-section and the
SBR cross-section. A system of 6 equations with 6 unknowns written in Eq. (9) was also numerically solved for discrete values of $k L$.

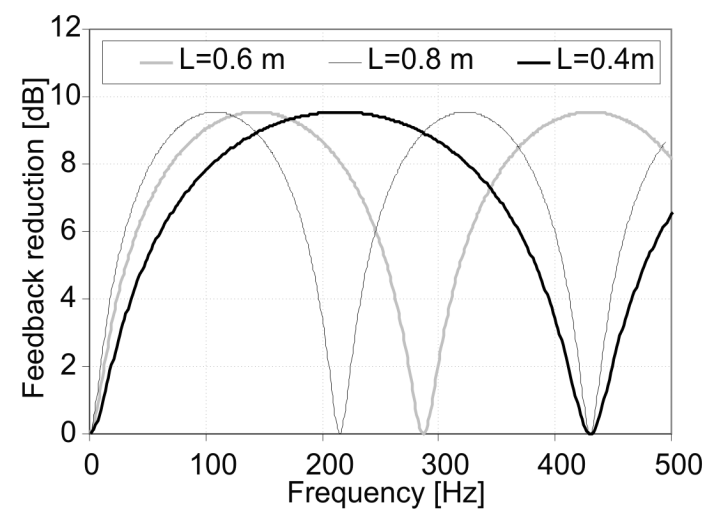

Fig. 3. A frequency spectrum of the feedback reduction for three different lengths of the side branch; cross section ratio $A_{1} / A_{2}$ was set to 8

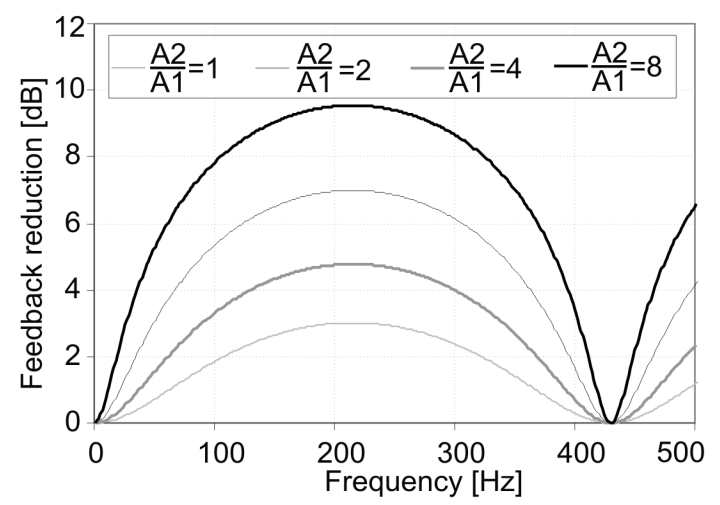

Fig. 4. A frequency spectrum of the feedback reduction for four different cross section ratios; a length of the side branch was set to $0.4 \mathrm{~m}$

The difference between the sound pressure level in an upstream direction and the sound pressure level in a downstream direction can be considered as a feedback reduction. In Figs. 3 and 4 , a feedback reduction for different geometries of the SBR is presented. In Fig. 3, a frequency response of feedback reduction is presented for different lengths of side branch resonator. The shorter the side branch resonator, the higher is the central frequency of feedback reduction and the broader is its useful frequency range. In Fig. 4 a frequency spectrum of feedback reduction is presented for different cross section ratios. $A_{1}$ presents the cross section of the main duct 


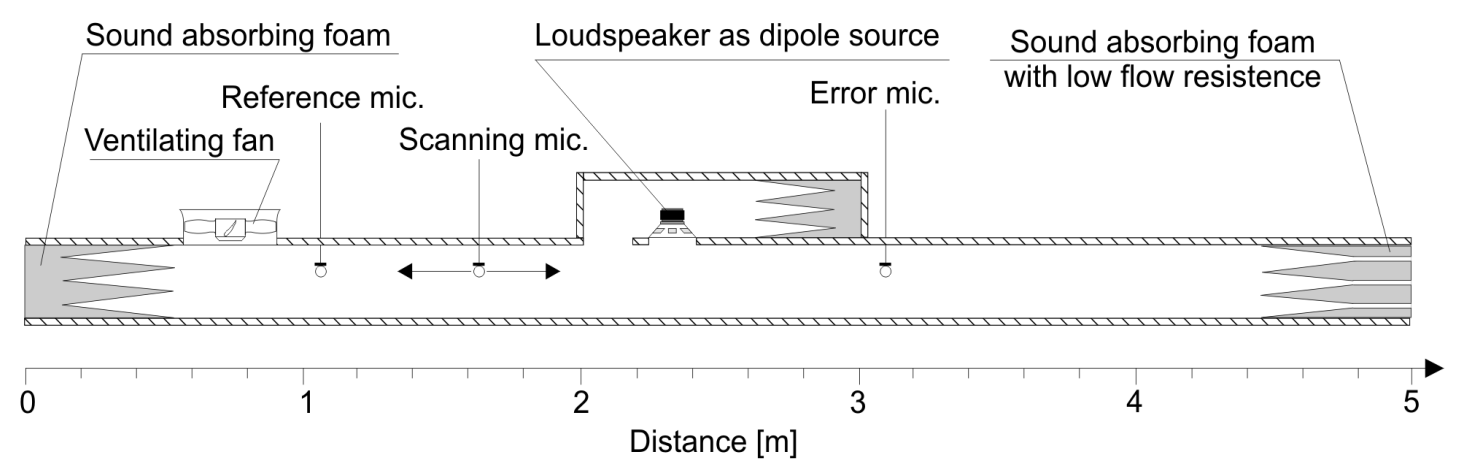

Fig. 5. Experimental setup

and $A_{2}$ presents the cross section of side branch resonator. Sound pressure, generated in the upstream direction, is proportional to $1 / A_{1}$. Sound pressure, generated in the downstream direction is proportional to $1 / A_{1}+1 / A_{2}$. The higher the ratio $A_{1} / A_{2}$, the better is the feedback reduction.

\section{EXPERIMENTAL SETUP AND MEASUREMENTS RESULTS}

The experimental setup is shown in Fig. 5. Geometry of the secondary source and damping in the SBR were determined according to the theoretical results. $L$ was shortened to $0.4 \mathrm{~m}$ and cross section ratio was set to $A_{1} / A_{2}=1$. SBR was partially filled with damping material, as shown in Fig. 5. The duct was made from $3 / 4$ " thick plywood. It was covered with hard plastic to ensure a low coefficient of sound absorption in the main duct.

The experimental set-up was built in order to measure a directivity of the new secondary source configuration, to determine the influence of damping in the SBR on the directivity, and to measure impulse responses of all acoustic paths and impulse responses of all electro acoustic elements.

A sound field in the duct was measured by a moving microphone. Panasonic omni directional Back Electret Condenser Microphone WM-61A was mounted on the pulley. The position of the microphone was controlled with a low-noise electric motor with a reduction gear system mounted on the duct using vibration insulation. The moving microphone was traversed with the speed of $1.5 \mathrm{~cm} / \mathrm{sec}$. Measurements were performed using a pink noise excitation. The pink noise was used as a test signal because it ensures a good signal to noise ratio in low-frequency range.

Measurement results of the sound pressure level field in the duct with anechoic termination are presented in Fig. 6. The sound pressure level field was generated by the secondary source with a SBR configuration as shown in Fig. 5. The sound field is not homogeneous. The secondary source has its own frequency response and a standing wave appears. The most important result is that the new secondary source generates much higher sound pressure levels to the right side (brighter spectrogram) than to the left (darker spectrogram).

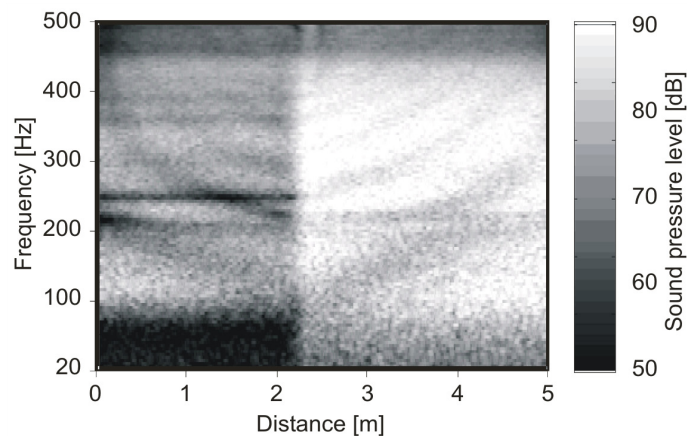

Fig. 6. Measured sound field in the duct, generated by the SBR secondary source; a duct had anechoic termination

A secondary path $S(n)$, a primary path $P(n)$, and a feedback path $F(n)$ were identified using impulse response measurement for different configurations of the secondary source. The secondary path and the feedback path impulse responses are presented in Figs. 7 and 8. These impulse responses are required for a valid and credible simulation of adaptive ANC system. 
Acoustic and electro-acoustic impulse responses used in ANC simulations were measured on real subsystems forming the HVAC model with an ANC system.

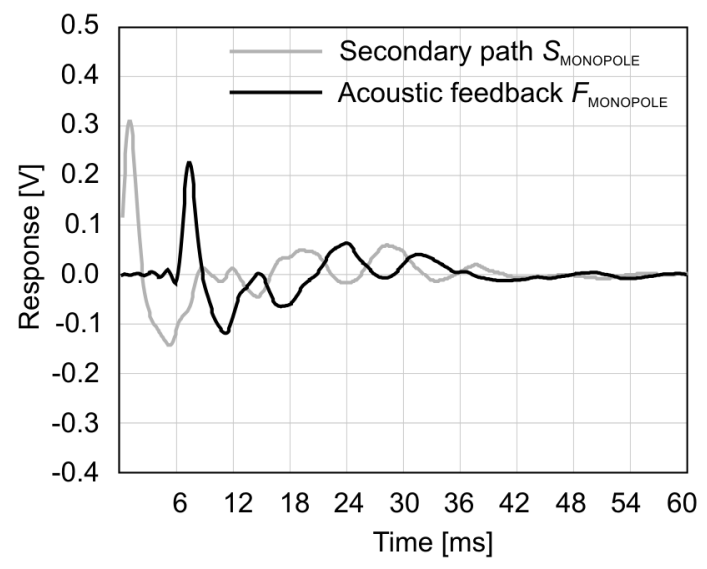

Fig. 7. Impulse responses of a secondary path and an acoustic feedback, measured with the classic monopole configuration of the secondary source

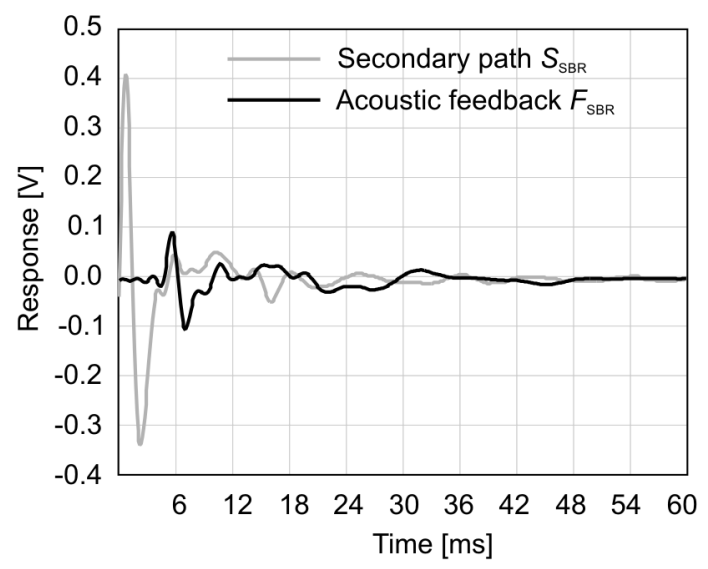

Fig. 8. Impulse responses of a secondary path and an acoustic feedback, measured with the new SBR configuration of the secondary source

The impulse response of the secondary path $S_{\text {MONOPOLE }}(n)$, obtained with the classical monopole secondary source is compared with the impulse response of the feedback path $F_{\text {MONOPOLE }}(n)$, obtained with the classical monopole secondary source in Fig. 7. The delay of the feedback is approximately $7 \mathrm{~ms}$. The amplitude of the feedback impulse is $2 / 3$ of the secondary path impulse. Acoustic feedback is significant.
The impulse response of the secondary path $S_{S B R}(n)$, obtained with the SBR secondary source is presented in Fig. 8, together with the impulse response of the acoustic feedback $F_{S B R}(n)$, obtained with the SBR secondary source. The impulse response of the secondary source with SBR configuration has a pronounced negative peak at $2 \mathrm{~ms}$. The ratio between the amplitude of the secondary path impulse response $S_{S B R}$ to the amplitude of the feedback impulse response $F_{S B R}$ is improved from 1.4 to 4.2. Acoustic feedback is three times smaller.

Impulse responses obtained with the classical monopole secondary source exhibit lowfrequency oscillations, which are more pronounced than in impulse responses obtained with the SBR secondary source. The SBR secondary source becomes stable much faster than the classical secondary source, and is therefore more suitable for control purposes. Also, a shorter FIR filter is needed for modeling the SBR secondary source than for modeling the classical secondary source.

One of the objectives of the secondary source design was to achieve better performance of the ANC system itself. Therefore, a few simulations of the ANC process were performed in order to determine the effects of the added SBR on the ANC process. Simulations of ANC were based on the measurement and analysis of the impulse responses. Simulations were performed with MathWorks Simulink. The adaptive LMS algorithm in a signal processing toolbox was rearranged in order to simulate the FX-LMS algorithm described in [9].

During the simulations of ANC for different secondary sources only the measured impulse responses representing different acoustical paths were changed in the model. The step size of the adaptive algorithm was accordingly adjusted. The maximum step size for the FX-LMS algorithm depends on the RMS of the reference signal. Therefore, simulations were performed with a maximum step size, which was determined for both configurations. When using a classic monopole as a secondary source, a maximum step size was $\mu_{\max , \text { MONOPOLE }}=0.000018$. When using the secondary source with the SBR configuration a maximum step size was $\mu_{\max , S B R}=0.000071$. All other settings in the model were kept constant. 
The results of ANC simulations are presented in Figs. 9 to 11. Two spectrograms of residual noise at error microphone position are presented in Figs. 9 and 10. In Fig. 9 a performance of the ANC system with the classical monopole secondary source is presented. In Fig. 10 a performance of the ANC system with the SBR secondary source is presented.

By comparing Fig. 9 with Fig. 10 it can be clearly seen that the convergence of the ANC system is faster with the SBR secondary source. Levels of residual noise after the finished convergence are also lower. Improvements can be seen in a broad frequency range. In comparison to the classical ANC system residual noise is higher only in the extremely low narrowband frequency range below $40 \mathrm{~Hz}$.

Four frequency spectra of primary noise and residual noise at error microphone position are presented in Fig. 11. A thick gray line presents primary noise spectra at the error microphone position if a classical monopole secondary source is attached to the duct. A thick black line presents primary noise spectra at the error microphone position if the SBR secondary source is attached to the duct. Even with the SBR secondary source switched off, some attenuation of primary noise is achieved at error microphone position. According to our predictions, primary noise is reduced around the SBR secondary source eigen-frequencies.
Insertion loss of the switched off SBR secondary source is depicted in Fig. 12. In a narrowband frequency range the insertion loss is over $3 \mathrm{~dB}$. It could be much higher, if less damping material would be inserted in the SBR. However, with less damping material in the SBR the directivity of the secondary source would not be achieved and acoustic feedback would reduce the performance of the ANC system.

Measurement results differ from pure theoretical expectations. The main reason for the deviation of the measured results from the theoretical ones is a significant amount of damping material, which was inserted in the SBR. In former studies [11] no damping was used in order to compare measurement results with the simulation results and to establish proper finite element model.

An additional reason for the deviation of the measured result from the theoretical one is that a loudspeaker acts like a mechanical resonator with own its mass, spring and damping. Furthermore, it has its own resonant frequency and acts like a membrane absorber. A resonant frequency of the loudspeaker used in the experiment was around $80 \mathrm{~Hz}$. Just around this frequency deviation of the measured results most significantly differs from the theoretical ones.

Two frequency spectra of residual noise at error microphone position with working ANC (thin

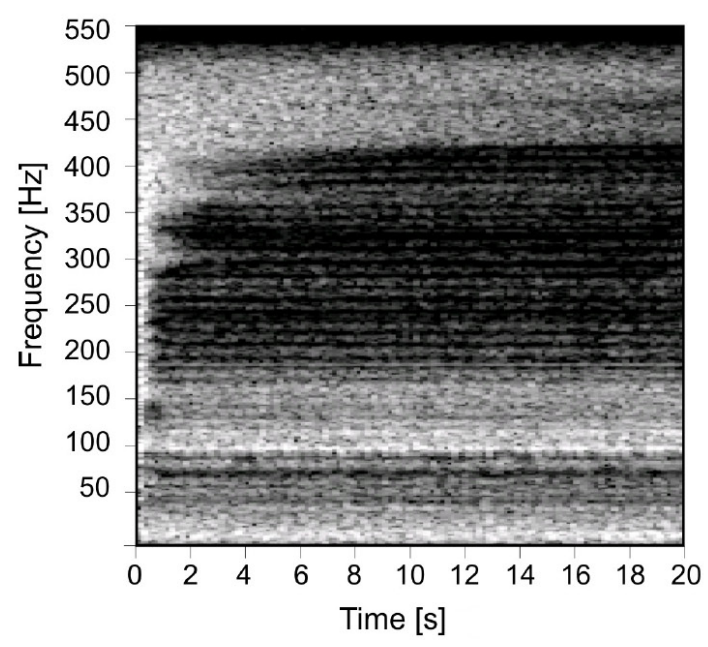

Fig. 9. Residual noise at the error microphone position obtained with an ANC system using a classical monopole secondary source

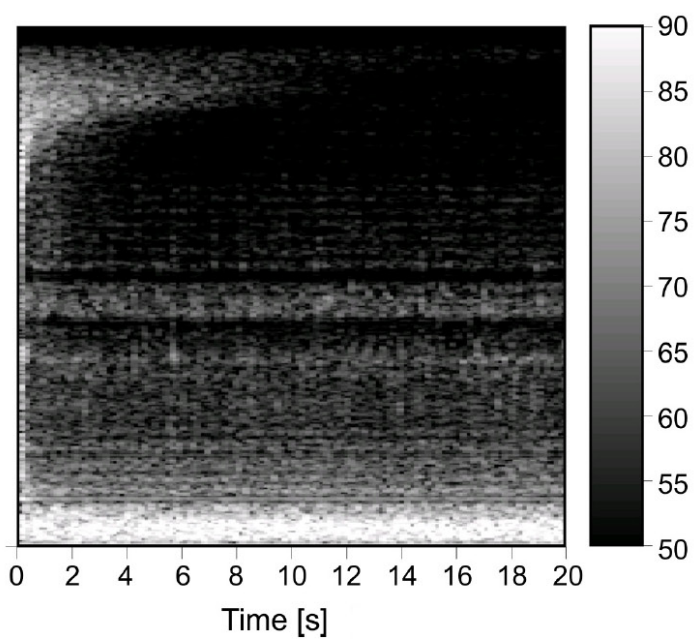

Fig. 10. Residual noise at the error microphone position, obtained with an ANC system using a secondary source with the SBR configuration 


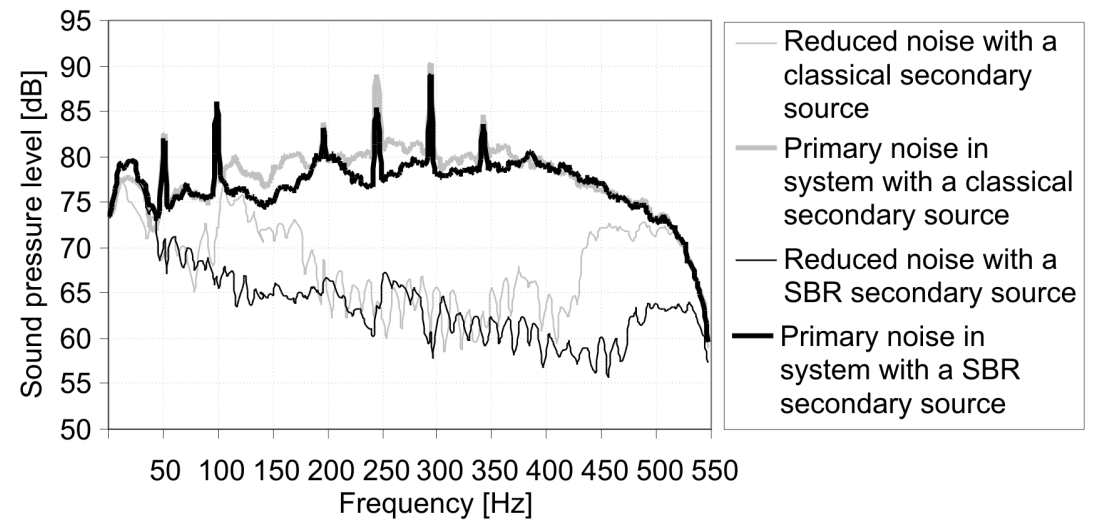

Fig. 11. Frequency spectra of ventilating fan noise and residual noise at error microphone position; with switched off ANC system (thick lines) and with switched on ANC system (thin lines); black lines for the $S B R$ secondary source and gray lines for the classical monopole source

lines) are also presented in Fig. 11. Both spectra present residual noise after the adaptive algorithm finished the convergence. A thin gray line presents residual noise spectra of ANC system using classical monopole secondary source. A thin black line presents residual noise spectra of ANC using the SBR secondary source. The ANC system with the SBR secondary source achieves lower residual noise in the broad frequency range. A frequency spectrum of the residual noise is smoother and an improvement is more than $5 \mathrm{~dB}$ in two frequency ranges (from 100 to $170 \mathrm{~Hz}$ and above $420 \mathrm{~Hz}$ ). Active noise control works much better if SBR secondary source is used.

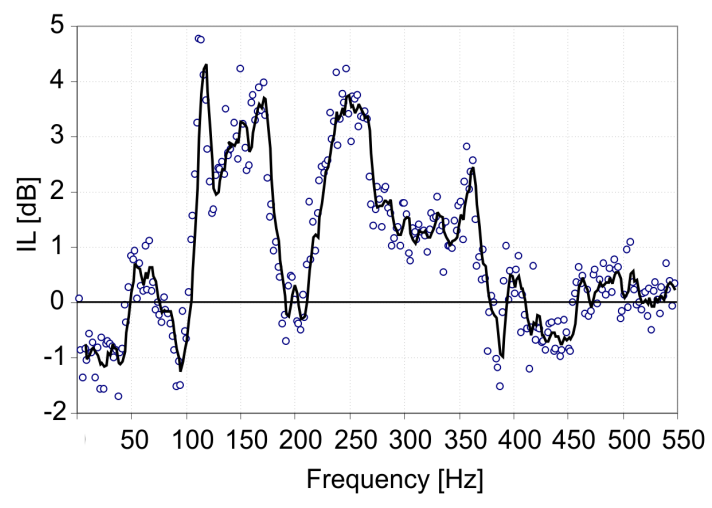

Fig. 12. Insertion loss of the switched off SBR secondary source

A sound pressure level across the experimental duct is presented in Fig. 13. It is presented for a frequency range around $120 \mathrm{~Hz}$. Results indicate to a standing wave which forms between the secondary and primary noise source due to the acoustic feedback. The node of this standing wave can be observed as a local minimum at $1.5 \mathrm{~m}$. This minimum $(-10 \mathrm{~dB})$ is pronounced only for the monopole type of the secondary source. When using the secondary source with the SBR configuration this minimum is only around $-3 \mathrm{~dB}$.

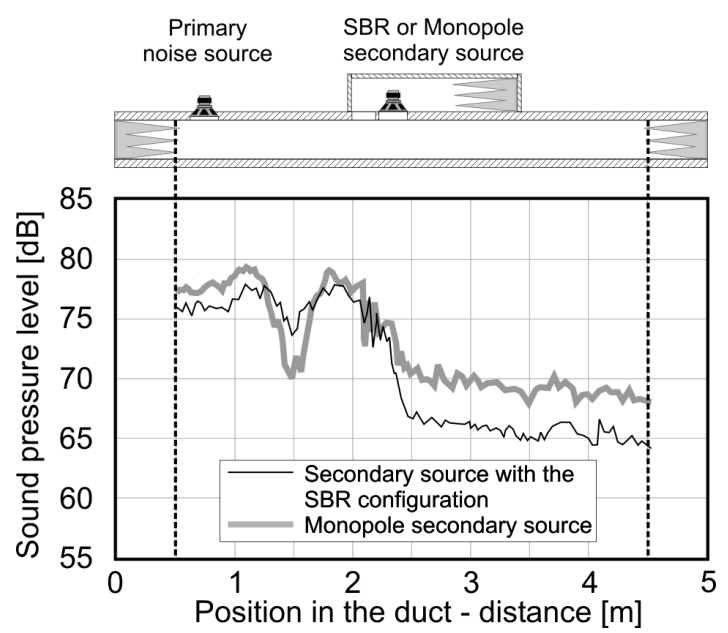

Fig. 13. Sound pressure level across the experimental setup

\section{CONCLUSIONS}

In this article it was demonstrated that a loudspeaker, working as a dipole source in a 
side branch resonator (SBR), can be used as a secondary source for ANC systems. The SBR has three different tasks in the proposed configuration. First, it prevents the acoustic short circuit in one direction. At the same time it allows an acoustic short circuit to happen in the other direction. Finally, the SBR acts like a resonator, enabling additional transmission loss of the system. This results in significant directivity of the secondary source and improved transmission loss in the desired frequency range. During the design process of the SBR secondary source special attention needs to be paid to proper selection of the SBR length, damping and opening cross section. Therefore, a simple analysis of the proposed configuration was carried out. The results of the analysis show that significant directivity can be achieved at low $k L$ values. This is in accordance with the purpose of active noise control to work in low frequency range. The distance between a loudspeaker and the opening from SBR should be short. A shorter distance between the loudspeaker and the SBR opening provides a flat frequency response of the secondary source on one hand, but reduces the efficiency of the secondary source to generate high sound pressure level on other.

Damping in the side branch resonator should be optimized. Too much damping reduces a SBR to work as a resonator. If there is no damping, the SBR secondary source would not have an unidirectional characteristic. Measurements of impulse responses enabled the simulations of the adaptive ANC. ANC simulations demonstrated that the ANC system with the SBR secondary source performs much better than the ANC system with the classical monopole secondary source. The acoustic feedback in feedforward ANC system is reduced when using the secondary source with SBR configuration. The convergence of the adaptive FX-LMS algorithm can be faster with the SBR secondary source, and residual noise is lower than with the classical monopole secondary source.

In future work a multi objective optimization could be performed. Optimization could involve a cross section ratio between the duct and the SBR, a damping, a distance between the loudspeaker and the SBR opening and a SBR length. After the optimization an additional increase of the system performance is expected.

\section{REFERENCES}

[1] Munjal, M.L. (1987). Acoustics of ducts and mufflers. John Wiley \& Sons, New York.

[2] Field, C.D., Fricke, F.R. (1998). Theory and applications of quarter wave resonators: A prelude to their use for attenuating noise entering buildings through ventilation openings. Applied Acoustics, vol. 53, no. 1-3, p. 117-132.

[3] Romeu, J., Saluena, X., Jimenez, S., Capdevila, R., Coll, L. (2001). Active noise control in ducts in presence of standing waves. Its influence on feedback effect. Applied Acoustics, vol. 62, p. 3-14.

[4] Geddes, E.R. (1994). Dual Bandpass Secondary Source, United States Patent, Patent No. 5,319, 165.

[5] Chen, K.T., Chen, Y.H., Hsueh, W.J., Wang, J.N., Liu, Y.H. (1998). The study of an adaptively active control on the acoustic propagation in a pipe. Applied Acoustics, vol. 55, p. 53-66.

[6] Swinbanks, M. (1973). The active control of sound propagation in long ducts. Journal of Sound and Vibration, vol. 27, no. 3, p. 411436.

[7] Tang, S.K., Cheng, J.S.F. (1998). On the application of active noise control in an open end rectangular duct with and without flow. Applied Acoustics, vol. 53, no. 1-3, p. 193210.

[8] Nelson, P.A., Elliot, S.J. (1995). Active control of sound. Academic Press, London.

[9] Kuo, S.M. (1996). Active Noise Control Systems, Algorithms and DSP implementation. Wiley intersc., New York.

[10] Prezelj, J., Čudina, M. (2007). Dipole like secondary source for restrain of acoustical feedback in active noise control systems. Internoise 2007, p. 479-489.

[11] Prezelj, J., Čudina, M. (2007). Dipole in orthogonal direction as a secondary source for active noise control in ducts. Acta Acustica united with Acustica, vol. 93, no. 1, p. 63-72.

[12] Tarabini, M., Roure, A., Pinhede, C. (2009). Active control of noise on the source side of a partition to increase its sound isolation. Journal of Sound and Vibration, vol. 320, no. 4-5, p. 726-743. 